\title{
Staging and natural history of cerebrovascular pathology in dementia
}

V. Deramecourt, MD, $\mathrm{PhD}$

J.Y. Slade, BSc

A.E. Oakley, MBiol

R.H. Perry, FRCPath

P.G. Ince, FRCPath

C.-A. Maurage, MD, $\mathrm{PhD}$

R.N. Kalaria, FRCPath

Correspondence \& reprint requests to Dr. Kalaria: raj.kalaria@ncl.ac.uk

Editorial, page 1032

Supplemental data at www.neurology.org
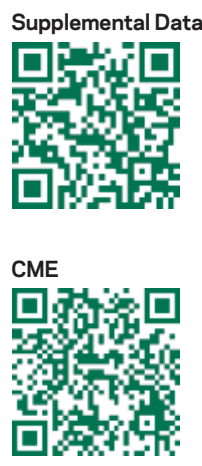

\section{ABSTRACT}

Objective: Most pathologic studies indicate that significant vascular changes are found in the majority of elderly persons, either alone or in association with neurodegenerative processes such as Alzheimer disease (AD) or dementia with Lewy bodies (DLB). Cumulative burden of cerebrovascular lesions can explain cognitive decline described as vascular cognitive impairment, but because there is a lack of consensus in the best way to quantify vascular pathology, the relationship between cognitive decline and cerebrovascular disease remains uncertain. We developed a rating scheme for cerebrovascular lesions using postmortem brains from patients with dementia from 2 European tertiary care memory clinics.

Methods: A total of 135 brains with a neuropathologic diagnosis of vascular dementia $(\mathrm{VaD})(\mathrm{n}=$ 26), $A D+\operatorname{VaD}(n=39), D L B+\operatorname{VaD}(n=21), A D+D L B+V a D(n=9), A D(n=19)$, and $D L B(n=$ 21) were investigated in this study. Cerebrovascular lesions were rated on large sections from the hippocampus, the temporal lobe, the frontal lobe, and basal ganglia.

Results: In patients with dementia, vessel wall modifications such as arteriolosclerosis or amyloid angiopathy are the most common and presumably the earliest changes. Modifications in perivascular spaces and myelin loss are the next most common. Lacunar or regional infarcts may occur as a consequence of an independent process or in the final phase of small vessel diseases.

Conclusion: A staging system based on this conceptual model of cerebrovascular pathology could enable the neuropathologic quantification of the cerebrovascular burden in dementia. Further studies are needed to determine whether this system can be used in large-scale studies to understand clinical-cerebrovascular pathologic correlations. Neurology ${ }^{\circledR}$ 2012;78:1043-1050

\section{GLOSSARY}

$\mathbf{A D}=$ Alzheimer disease; $\mathbf{C V L}=$ cerebrovascular lesions; $\mathbf{D L B}=$ dementia with Lewy bodies; $\mathbf{L F B}=$ Luxol fast blue; $\mathbf{N B T R}=$ Newcastle Brain Tissue Resource; SVD = small vessel disease; $\mathbf{V a D}=$ vascular dementia; $\mathbf{V C l}=$ vascular cognitive impairment; $\mathbf{W M}=$ white matter

Vascular cognitive impairment (VCI) is regarded as the second most common cause of cognitive disorder after Alzheimer disease (AD). ${ }^{1} \mathrm{VCI}$ is a frequent consequence of various cerebrovascular lesions (CVL) resulting from disrupted circulation or perfusion in different brain regions. Imaging and postmortem studies have shown that CVL may also be found in cognitively normal elderly subjects ${ }^{2-4}$ and in more than $50 \%$ of cases with neurodegenerative disorders such as AD or dementia with Lewy bodies (DLB). ${ }^{5}$

Despite considerable efforts, to date there are no consensual neuropathologic criteria for vascular $(\mathrm{VaD})$ and mixed dementia. The postmortem diagnosis of VCI mostly relies on the identification of significant $\mathrm{CVL}^{6}$ in the absence of other changes that may explain the cognitive decline. ${ }^{7}$ For mixed cases, neuropathologists are compelled to identify a threshold above which CVL would be considered as significant from a strictly subjective point of view. Such quantification could only be obtained from sampled brain areas since an extensive microscopic examination of the whole brain is impractical. Attempts have been made to identify

From the Institute for Ageing and Health (V.D., J.Y.S., A.E.O., R.H.P., R.N.K.), Campus for Ageing and Vitality, Newcastle University, Newcastle upon Tyne, UK; Memory Clinic and Histology Department (V.D.) and Pathology and Histology Department (C.-A.M.), Univ Lille Nord de France, Lille University Hospital, Lille, France; and The Academic Unit of Pathology (P.G.I.), Medical School, University of Sheffield, Sheffield, UK. Study funding: Funding information is provided at the end of the article.

Disclosure: Author disclosures are provided at the end of the article. 
Table 1 Demographic and pathologic features of the cohort

\begin{tabular}{|c|c|c|c|c|c|c|c|}
\hline & $\mathrm{VaD}$ & $A D+V a D$ & $\begin{array}{l}\mathrm{AD}+\mathrm{DLB}+ \\
\mathrm{VaD}\end{array}$ & $\mathrm{DLB}+\mathrm{VaD}$ & $A D$ & DLB & Total \\
\hline No. & 26 & 39 & 9 & 21 & 19 & 21 & 135 \\
\hline$M / F$ & $13 / 13$ & $16 / 23$ & $5 / 4$ & $12 / 9$ & $9 / 10$ & $13 / 8$ & $68 / 67$ \\
\hline Age at death, $y$, mean $\pm S D$ & $81.2 \pm 7.5$ & $83.9 \pm 4.9$ & $81.6 \pm 3.9$ & $79.7 \pm 5.4$ & $78.4 \pm 10.3$ & $77.5 \pm 5.8$ & $80.8 \pm 6.9$ \\
\hline $\begin{array}{l}\text { Braak stage for neurofibrillary } \\
\text { pathology }(0-6) \text {, median (range) }\end{array}$ & $2(1-3)$ & $6(4-6)$ & $6(4-6)$ & $2(1-3)$ & $6(5-6)$ & $2(1-3)$ & $4(1-6)$ \\
\hline $\begin{array}{l}\text { CERAD score for amyloid plaques } \\
(0-3) \text {, median (range) }\end{array}$ & $2(0-3)$ & $3(2-3)$ & $3(2-3)$ & $2(1-3)$ & $3(2-3)$ & $2(0-3)$ & $2(0-3)$ \\
\hline \multicolumn{8}{|l|}{ Lewy body pathology, $n$} \\
\hline Brainstem predominant & 2 & 1 & 0 & 0 & 3 & 0 & 6 \\
\hline Limbic & 0 & 0 & 5 & 5 & 0 & 2 & 12 \\
\hline Neocortical & 0 & 0 & 4 & 16 & 0 & 19 & 39 \\
\hline Vascular score $(0-20)$, mean \pm SD & $14.4 \pm 2.0$ & $13.2 \pm 1.9$ & $13.0 \pm 1.4$ & $12.1 \pm 1.8$ & $7.4 \pm 1.8$ & $6.8 \pm 2.5$ & $11.5 \pm 3.5$ \\
\hline
\end{tabular}

Abbreviations: $A D=$ Alzheimer disease; CERAD = Consortium to Establish a Registry for Alzheimer's Disease; DLB = dementia with Lewy bodies; $\mathrm{VaD}=$ vascular dementia.

such threshold values but the drawback is that such evaluation becomes a complex tedious procedure. ${ }^{8}$

To further the National Institute of Neurological Disorders and Stroke-Canadian Stroke Network VCI harmonization standards guidelines ${ }^{6}$ and our previously proposed classification of vascular pathology, ${ }^{7}$ we investigated 135 brains with various degrees of CVL burden. The main goal of our study was to conceptualize the natural history of CVL and operationalize it into a cerebrovascular staging system.

METHODS Selection of subjects, brain sampling, and histopathologic procedures. Cases were obtained from the Newcastle Brain Tissue Resource (NBTR, UK) and the Lille University Hospital brain bank (Lille Neurobank, France). All patients had dementia prior to death and were previously clinically followed up in tertiary care memory clinic settings either at the Newcastle General Hospital or the Lille University Hospital. Because we have no information on patients with dementia who died and did not undergo brain autopsy, it is unknown whether our series over- or under-recruited persons with cerebrovascular pathology. We included 135 cases (died between 1990 and 2010) in this study: 109 cases from NBTR and 26 from Lille Neurobank. Based on the final neuropathologic diagnosis, they consisted of $26 \mathrm{VaD}, 39 \mathrm{AD}+\mathrm{VaD}, 9 \mathrm{AD}+\mathrm{DLB}+\mathrm{VaD}, 21$ $\mathrm{DLB}+\mathrm{VaD}, 19 \mathrm{AD}$, and $21 \mathrm{DLB}$ cases.

Table 1 provides the demographics and the pathologic features of the cases. Autopsies were performed within 12-36 hours after death. Upon gross examination of the whole brain, vascular or other lesions were noted. In the majority of the cases, only the left hemispheres were fixed in formalin for $4-8$ weeks. If any visible macroscopic infarcts or lacunes were observed on the right side the whole coronal slice was also fixed. ${ }^{9}$ There were no differences in the length of fixation between the groups. The neuropathologic diagnoses of $\mathrm{VaD}, 7 \mathrm{AD},{ }^{10}$ and $\mathrm{DLB}^{11}$ were made by thorough histopathologic examination of extensively sampled brain sections. The diagnosis of mixed pathologies, i.e., association of significant CVL with neurodegenerative lesions, was based on the assessment of 4 experienced investigators (R.H.P., P.G.I., R.N.K., and C.A.M.) at different times.

All cases had available paraffin-embedded tissue blocks from frontal and temporal lobes, hippocampus, and basal ganglia. These regions were chosen because they represent relevant cerebral systems involved in cognition and receive blood from each major cerebral arterial supply. The sampling of brain involved relatively larger sections (average size $6 \times 5 \mathrm{~cm}$ ) as is normal practice in Newcastle and Lille but not necessarily the case in many laboratories. Ten-micrometer-thick paraffin-embedded tissue sections were cut from the frontal lobe at the level of the olfactory bulbs, the temporal lobe at the level of the anterior hippocampus, and basal ganglia (lenticular nucleus and anterior thalamus) at the level of mamillary body, and stained for hematoxylin-eosin and Luxol fast blue (LFB). To minimize variability in LFB staining, control sections were included, and tissue sections were prepared by the same technician in both centers and stained with or using freshly prepared tinctorial and buffer solutions.

Assessment of CVL. Following previously identified guidelines to major lesions, ${ }^{7} \mathrm{CVL}$ were rated in the temporal and frontal lobes as follows: arteriolosclerosis (concentric hyaline thickening of small arteries, from 40 to $150 \mu \mathrm{m}$ in diameter, leading to a stenosis of the vessel lumen), amyloid angiopathy, perivascular hemosiderin leakage, perivascular spaces dilatation in the deep and juxtacortical white matter (WM), myelin loss, and cortical micro $(<0.5 \mathrm{~cm})$ and large infarcts $(>0.5 \mathrm{~cm})$. In the hippocampus, we rated the neuronal loss, perivascular spaces dilatation, and the presence of micro and large infarcts. In the basal ganglia, we assessed arteriolosclerosis, perivascular spaces dilatation, the density of microinfarcts, and large infarcts, either lacunar (defined as cavitating infarcts measuring up to $15 \mathrm{~mm}$ ) or territorial.

Except for cortical and hippocampal infarcts which were numbered on each slide, each lesion was graded according to severity using a semiquantitative scale ranging from 0 to $3(0=$ normal, $1=$ mild changes, $2=$ moderate lesions, and $3=$ severe modification; additional written definitions for this scale are available in table e-1 on the Neurolog ${ }^{\circledR}$ Web site at www. neurology.org). The degrees of changes were based on the worst 
0

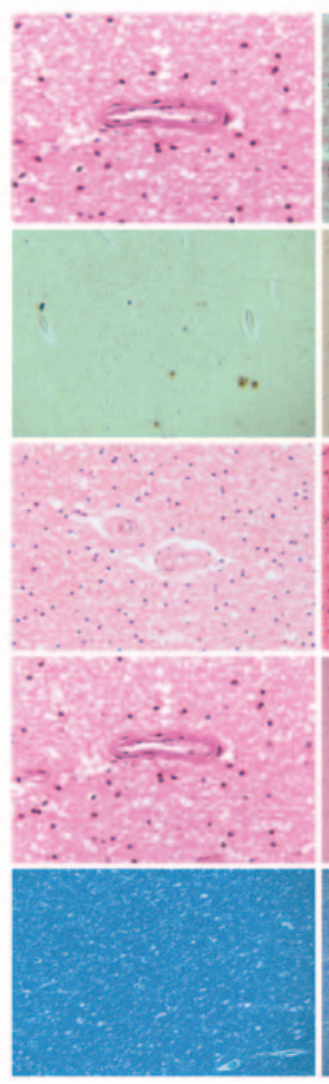

\section{Perivascular hemosiderin leakage}

Myelin loss
1

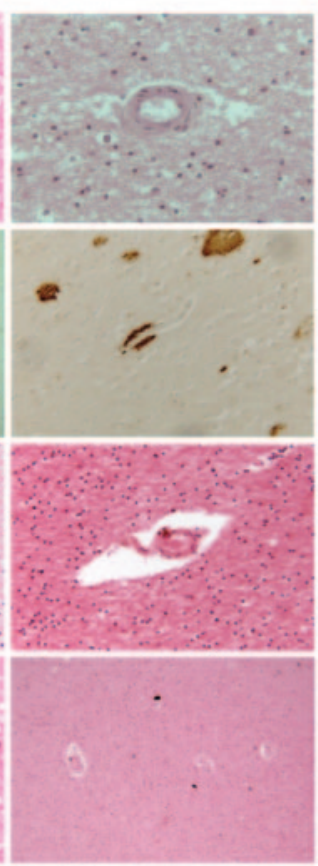

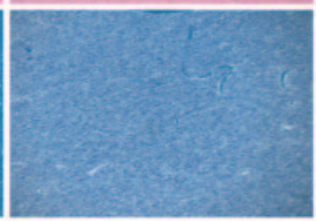

2
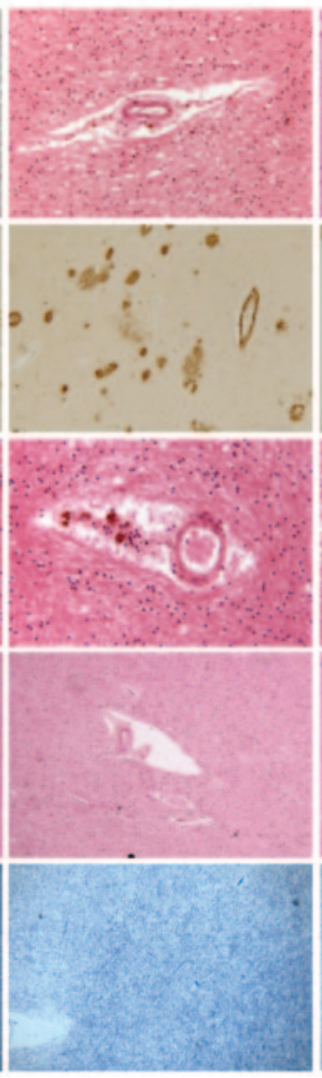

3
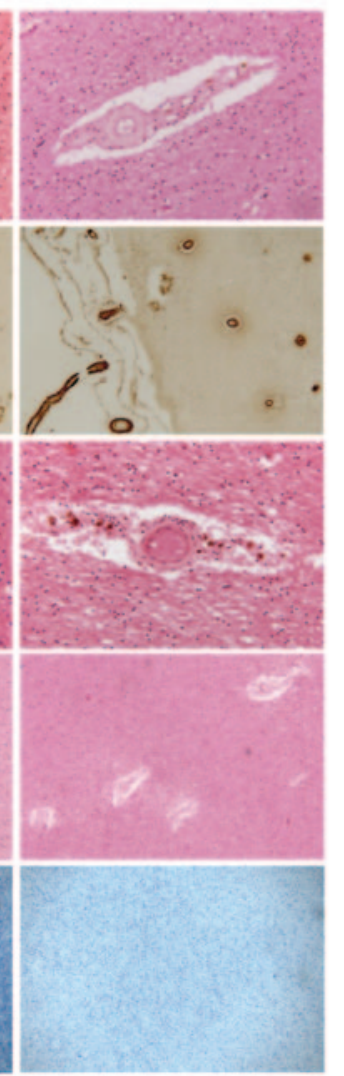

lesion in the section. In the current analyses, mild changes were not considered in the abnormal category. Some representative examples are shown in figure 1. Acute terminal ischemic or hemorrhagic lesions were not taken into account. The CVL were assessed by the same neuropathologist (V.D.), blinded to the final diagnosis. However, in a sample of 40 tissue sections containing various degrees of CVL burden, the agreement between 2 assessors (V.D. and R.K.) was above $90 \%$ for each CVL subtype.

Standard protocol approvals, registration, and patient consents. The clinical study had ethical approval from the local Newcastle and Lille ethics committees and all participants gave written consent to brain tissue donation. Use of brain tissue was also approved by the local research ethics committee of the Newcastle upon Tyne Hospitals NHS Foundation Trust, the NBTR committee, and the Lille University Hospital ethics committee.

RESULTS CVL frequency. In this descriptive presentation of CVL frequency, we only considered moderate to severe modifications (semiquantitative score $\geq 2$ ). In the frontal lobe, arteriolosclerosis was present in $83.7 \%$ of cases. Amyloid angiopathy was observed in $31.1 \%$. Overall, $89.6 \%$ of the cases had frontal localization of small vessel disease (SVD). Deep WM perivascular spaces dilatation was observed in $79.3 \%$, and in $63 \%$ of cases in the juxtacortical WM. Frontal myelin loss was present in $63 \%$ of the cases. Frontal cortical microinfarcts were found in $28.9 \%$ of the cases and $8.1 \%$ had frontal large infarcts.
In the temporal lobe, arteriolosclerosis was observed in $80.7 \%$ of the cases, and amyloid angiopathy in 37\%. Overall, $88.1 \%$ had temporal localization of SVD. Deep WM perivascular spaces dilatation was present in $77.8 \%$ of the cases, and in $64.4 \%$ in the juxtacortical WM. Temporal myelin loss was present in $48.9 \%$ of the cases. Temporal cortical microinfarcts were seen in $28.1 \%$ and $6.7 \%$ had temporal large infarcts.

In the hippocampus, perivascular spaces dilatation was observed in $48.1 \%$ of the cases. Microinfarcts were present in $17.8 \%$, and large infarcts were observed in $3.7 \%$.

In the basal ganglia, arteriolosclerosis was present in $89.6 \%$ of the cases. Perivascular space dilatation was observed in $68.1 \%$. Microinfarcts were present in $37 \%$, and large infarcts in $11.9 \%$. Figure 2 illustrates the CVL frequency and the comparisons between pathologic subgroups.

From the distribution of CVL to the formulation of a vascular staging system. Based on these observations, we considered a hypothesis based on the relative burden of CVL throughout the brain. The most common lesions were vessel wall modifications (arteriolosclerosis, amyloid angiopathy, or both), followed by perivascular 
Temporal lobe

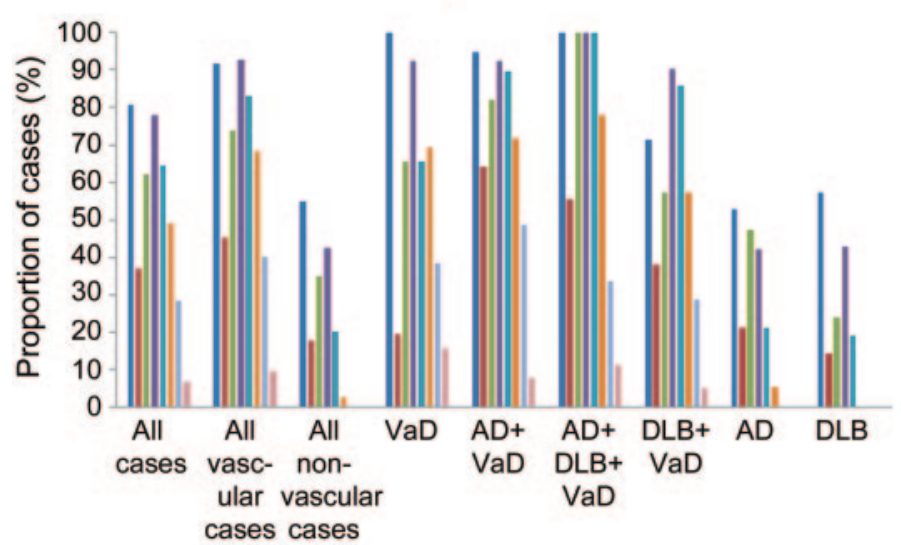

Frontal lobe

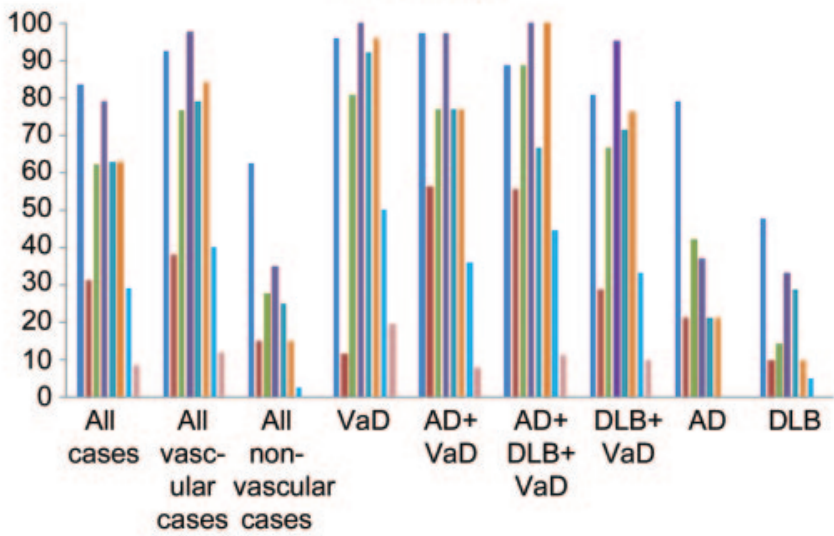

\begin{abstract}
= Arteriolosclerosis = Amyloid angiopathy

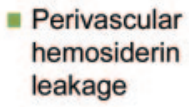

- Perivascular hemosiderin leakage

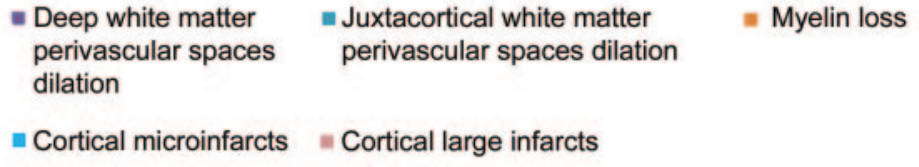

Basal ganglia

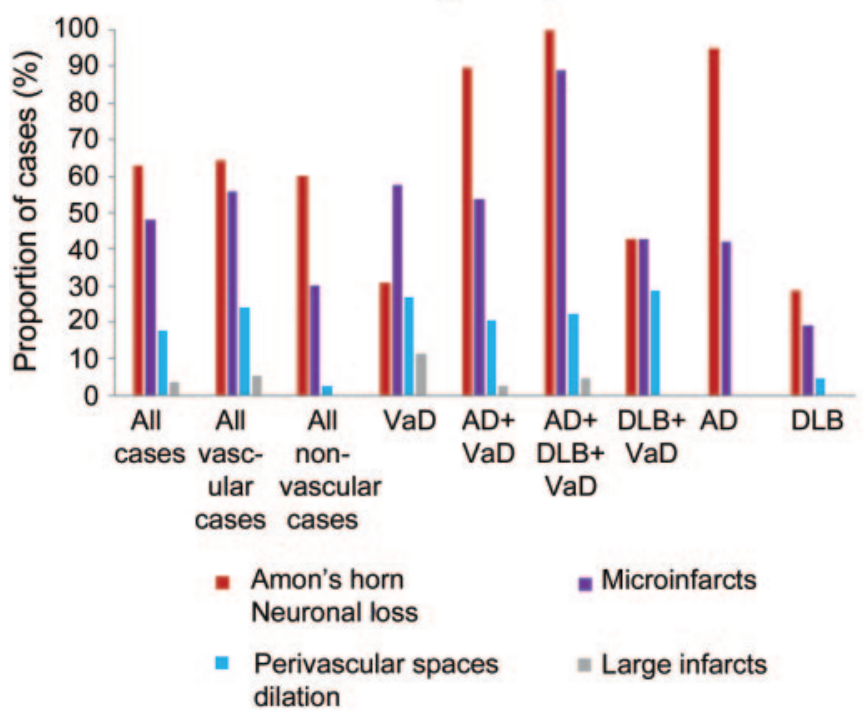

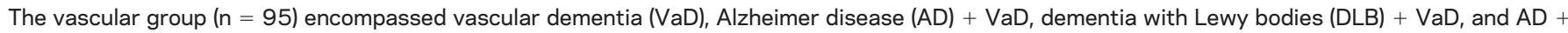

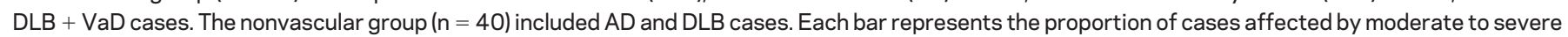
modifications or by the presence of infarcts.

space modifications (hemosiderin leakage, perivascular space dilatation), myelin loss, and, least commonly, infarcts. To test our hypothesis, we classified the $135 \mathrm{pa}-$ tients according to the frequency distribution of each type of CVL. We found a roughly pyramidal-shape distribution of CVL in each brain region we investigated. The classification obtained for the frontal lobe is illustrated in figure 3. In each brain region, with only a few exceptions, we observed a similar hierarchy in the distribution of CVL.

Among the 11 cases with a large infarct in the frontal lobe, 8 also had microinfarcts in the same lobe. Eighty-three percent of the cases with large or microinfarcts in the frontal lobe also had myelin loss in the frontal lobe. All subjects except one who had significant myelin loss in the frontal lobe also had perivascular space dilatation, either in the deep or the juxtacortical WM. Finally, all the cases with infarcts, myelin loss, or perivascular space dilatations in the frontal lobe had vessel wall modifications such as arteriolosclerosis, amyloid angiopathy, or both. Similar results were found in the temporal lobe. In the basal ganglia, all cases but one with one large/ lacunar infarct also had microinfarcts. All cases 
Figure 3 Distribution of the cerebrovascular lesions, example of the frontal lobe

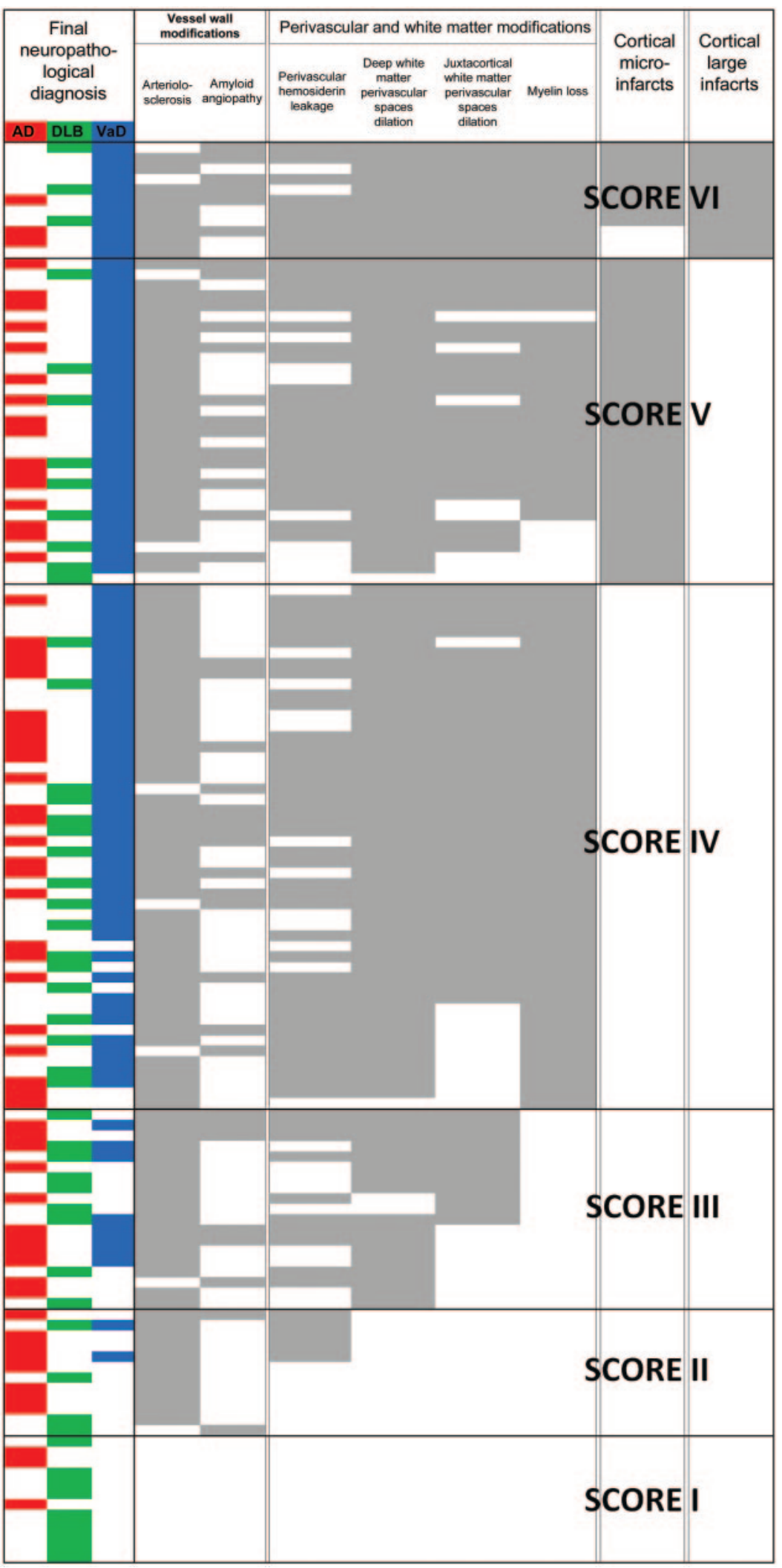

Cases were ordered according to the severity of cerebrovascular lesion (CVL) assessment in the frontal lobe. Gray-shaded areas represent moderate to severe modification or the presence of infarcts. The progressive increment of CVL was weighted into 7 scores from 0 to $\mathrm{VI}$ as defined in table 2. A similar hierarchical extent was observed in the temporal lobe and basal ganglia (not shown). $A D=$ Alzheimer disease; $\mathrm{DLB}=$ dementia with Lewy bodies; $\mathrm{VaD}=$ vascular dementia. with ischemic lesions had perivascular spaces dilatations and arteriolosclerosis.

We further made our model more operational, and translated it into a global vascular score. We divided the history of CVL into 7 stages for the temporal and the frontal lobes ( 0 to $\mathrm{VI}$ ) and 4 stages for the hippocampus and basal ganglia (0 to IV), as described in table 2 . A vascular score was calculated by adding the 4 previous stages (maximum score $=20$ ). In whole series, the vascular scores ranged from 3 to 19 , with a median score of 12 . As expected, 94 out of the 95 cases $(98.9 \%)$ that were considered as mixed or pure $\mathrm{VaD}$ according the previous standard neuropathologic assessment had a vascular score $\geq 10$. Of the 40 cases considered as pure neurodegenerative dementia according to previous standard neuropathologic examination, 37 (92.5\%) had a score $<10$. Thus, our staging system replicated our prior qualitative diagnostic impressions.

DISCUSSION Our aim was to transform a conceptual model of CVL into an operational staging system for use in evaluating late-life cognitive disorders. We performed an extensive inventory and evaluate the frequency of CVL in a large series of postmortem brains from longitudinally followed patients with dementia and from 2 European brain banks. Since the definitions of CVL are often institution-specific and of variable quality, ${ }^{12}$ we chose to quantify those with most unequivocal description: modifications of small vessel wall (arteriolosclerosis and amyloid angiopathy), perivascular spacing, myelin loss in the WM, and infarcts. The latter were subdivided by size into microinfarcts (not visible on gross examination) and large infarcts (either lacunar or territorial). These lesions were also selected according to their clinicopathologic relevance in VCI. ${ }^{6}$ Infarcts and WM changes have long been associated with cognitive impairment. ${ }^{13}$ The clinical relevance of perivascular spaces dilatations is still controversial, but these modifications are associated with age, blood pressure, and MRI of SVD. ${ }^{14}$ Perivascular hemosiderin leakage has recently been shown to represent a neuropathologic marker of vascular frailty in dementia, linked to both amyloid angiopathy and vascular risk factors. ${ }^{15}$

Our study confirms the high frequency and the widespread heterogeneity of CVL among brains from patients with dementia, as published in previous postmortem cohorts. ${ }^{2,16,17}$ Our systematic semiquantitative scoring provides a precise overview of the relative frequency of each CVL across 4 strategic regions in various conditions defined by routine neuropathologic examination. The most frequent CVL were arteriolosclerosis and enlarged perivascular spaces. Arteriolosclerosis was largely predominant in 


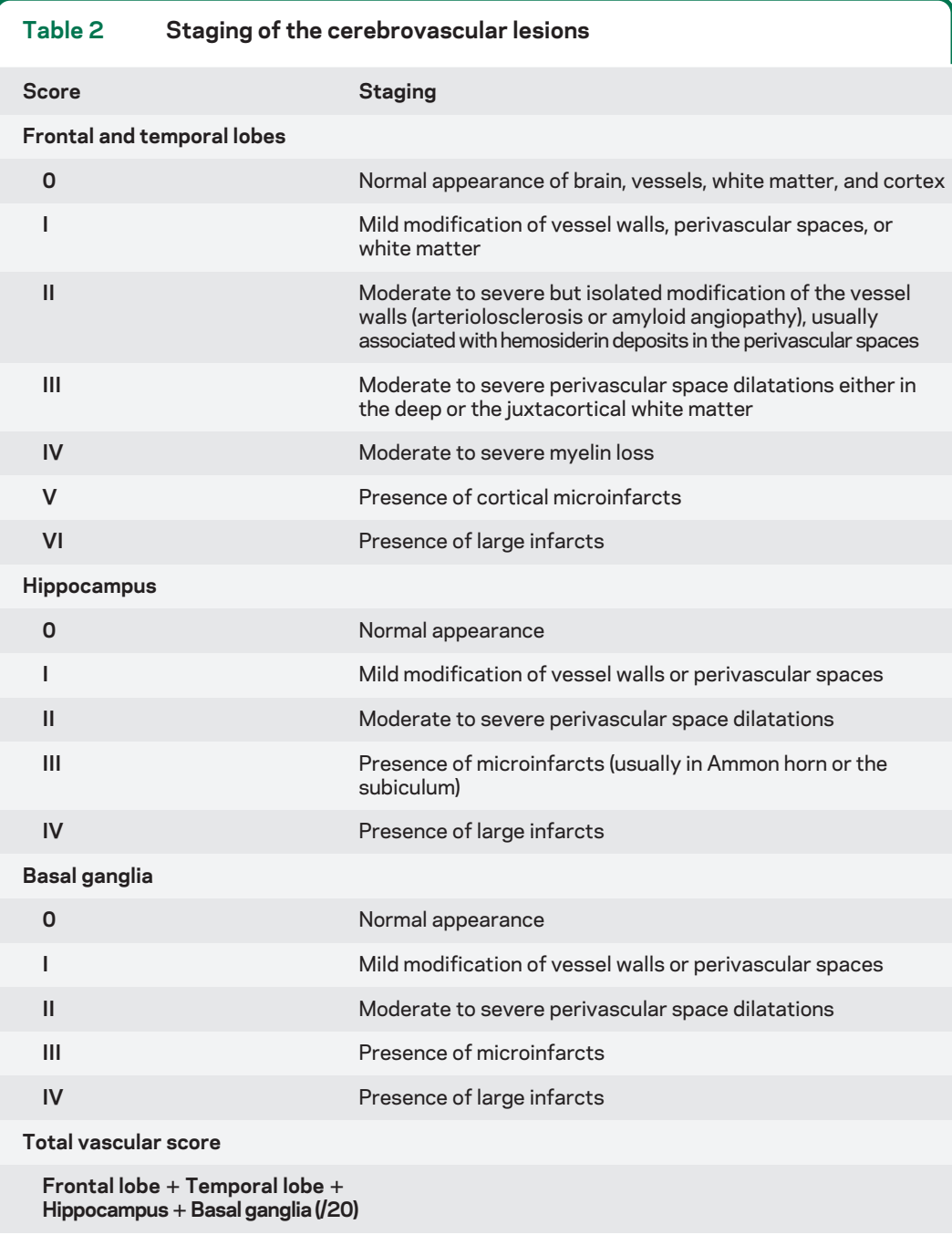

the basal ganglia. CVL were more frequent in the temporal lobe from mixed cases (AD or DLB + $\mathrm{VaD}$ ) than the $\mathrm{VaD}$ cases, suggesting that the neurodegenerative process by itself may contribute to WM damage in this location. ${ }^{18}$ Conversely, CVL were more frequent in the frontal lobe in $\mathrm{VaD}$ cases than mixed cases. Hippocampal microinfarcts were more frequent in this (17.8\% of all cases) than those in a recently reported cohort. ${ }^{19}$

The analysis did not address the contribution to cognitive impairment by the burden of cerebrovascular pathology. However, the development of a grading scheme for CVL that reflects sequential worsening of pathologic changes is necessary antecedent to understanding clinical-pathologic correlations. Our demonstration of the spectrum of pathologic changes ranging from very common to less common shows that there may be a quantitative way of representing CVL. Initial lesions are vessel wall modifications: arteriolosclerosis, amyloid angiopathy, or a combination of both. These 2 types of SVD, even if they are completely different in nature, are both linked to parenchyma damage, either WM changes or cortical microinfarcts,, $50-22$ although their precise pathogenetic mechanisms are unclear. ${ }^{16,23}$ Modifications of perivascular spaces could represent early consequences of altered vessel wall permeability in arteriolosclerosis, leading to hemosiderin leakage and alteration in the perivascular parenchyma. ${ }^{24}$ Hypoperfusion may then explain modifications and dilatation of the perivascular spaces, myelin loss in the WM. Cortical or subcortical microinfarcts and large infarcts seem to be the latest events in the time course of cerebral SVD. However, as illustrated by our rare cases with cortical microinfarcts but without vessel wall modification or WM damage, systemic microemboli represent an alternative etiology of small ischemic cortical lesions. Based on this distribution, we devised a scoring system for cerebral SVD with a special focus on microscopic lesions. Our vascular score, defined by the addition of the SVD stages in the 4 regions of interest, was in good agreement with the previous standard neuropathologic assessment of cerebrovascular pathology in these cases.

Our study has limitations. The fact that our assessment of CVL was only performed on a limited but practicable number of sections, i.e., 8 per brain (4 regions stained twice), could represent a critical constraint. However, rather than aiming at an extensive numbering of lesions, which may be neither practicable nor more informative, we focused on the hierarchy of the pathologic processes in each major arterial territory of the brain. Alternate sampling was not available in this retrospective postmortem cohort which thus does not entirely comply with the harmonization standards. ${ }^{6}$ However, in SVD, microscopic CVL are usually symmetrically distributed ${ }^{25,26}$ as even judged by the examination of whole-brain sections to reveal all the lesions and their extent. ${ }^{27}$ In our more recent study on poststroke stable and dementia cases there was also no major impact on laterality of medial temporal atrophy or hippocampal microscopic changes. ${ }^{28}$ In agreement with the harmonization standards, ${ }^{6}$ we recommend that alternate sampling should be now widely used in prospective cohorts. We recognize, however, that even the limited number of brain regions we studied may exceed available resources on a clinical neuropathologic service. Unfortunately, cerebrovascular pathology requires more intensive histologic examination. Second, the staging mostly relies on the natural history of arteriolosclerosis and atherosclerosis and may not reflect other causes of vascular injuries of the brain. Cardioembolic or hemodynamic infarcts may not perfectly fit with the hierarchical extent we describe. Nevertheless, the vast majority of cases with VCI are, at least in part, in causal relationship with 
arteriolosclerotic SVD. Pure cardioembolic or hemodynamic $\mathrm{VaD}$ cases are rare and should be considered separately. Finally, we did not include brains from aged controls without dementia and were not able to compare the distribution of CVL between cognitively impaired and nonimpaired groups. The extent of overlap in vascular scores between cognitively normal persons and those with dementia could be considerable. ${ }^{29,30}$

The vascular scores proposed here as an index for the vascular burden are presented to the clinicians, pathologists, and dementia researchers who called for a long time for a relevant way to assess cerebrovascular pathology despite several factors preventing the creation of neuropathologic criteria for $\mathrm{VaD} .{ }^{31}$ Replication of our studies worldwide is needed to assess if the staging system is useful, since the lack of adherence was one of the reasons explaining why none of the previous published criteria for $\mathrm{VaD}$ were widely accepted. However, our procedure relies on routine examination of the brain and does not necessarily require extensive sampling or supplemental or time-consuming techniques. Similar assessment in a cognitively unimpaired, agematched sample of subjects would represent an important addition to the literature.

Our proposed scheme of cerebral SVD reflects that there is probably not a clear cutoff threshold between cases with significant CVL burden and cases with pure neurodegenerative diseases. On the contrary, cerebral SVD more reliably represents a spectrum from initial vessel wall modifications to the later occurrence of WM damage and cortical infarcts. Further clinical pathologic studies are required to assess the clinical relevance of evaluating the CVL burden for diagnostic practice or research purposes.

\section{AUTHOR CONTRIBUTIONS}

Dr. Deramecourt: draft/revision of the manuscript, study design, analysis/ interpretation of the data, acquisition of the data. J.Y. Slade: acquisition of the data. A.E. Oakley: acquisition of the data. Dr. Perry: acquisition of the data. Dr. Ince: acquisition of the data. Dr. Maurage: acquisition of the data. Dr. Kalaria: draft/revision of the manuscript, study design, analysis/ interpretation of the data, acquisition of the data, study supervision, obtaining funding.

\section{ACKNOWLEDGMENT}

The authors thank the patients and families for cooperation in this study and Dr. Tuomo Polvikoski (IAH) for facilitating provision of some of the Newcastle cases.

\section{STUDY FUNDING}

Supported by grants from the UK Medical Research Council (MRC, G0500247), Newcastle Centre for Brain Ageing and Vitality (BBSRC, EPSRC, ESRC and MRC, LLHW), the Alzheimer's Research (ARUK), and the National Institutes of Health (NINDS). Tissue for this study was provided by the Newcastle Brain Tissue Resource, which is funded in part by a grant from the UK MRC (G0400074), by the Newcastle NIHR Biomedical Research Centre in Ageing and Age Related Diseases award to the Newcastle upon Tyne Hospitals NHS Foundation Trust, and by a grant from the Alzheimer's Society and ARUK as part of the Brains for Dementia Research Project. V.D. received a grant from the Journe'es de Neurologie de Langue Française society.

\section{DISCLOSURE}

Dr. Deramecourt, J.Y. Slade, A.E. Oakley, and Dr. Perry report no disclosures. Dr. Ince serves on scientific advisory boards for Medical Research Council UK Neurosciences and Mental Health Board; serves as Executive Editor for Neuropathology and Applied Neurobiology and for Clinical Neuropathology and on the editorial boards of Journal of Neuropathology and Experimental Neuropathology and Dementia and Geriatric Behavioral Disorders; and receives research support from Medical Research Council UK and Alzheimer Research UK. Dr. Maurage reports no disclosures. Prof. Kalaria serves on the editorial board of European Neurology, Alzheimer's Disease and Associated Disorders, Neuropathology and Applied Neurobiology, NeuroReport and Behavioral and Brain Functions; has received speaker honoraria from Pfizer Inc; and has received research support from the Alzheimer's Research Trust.

Received March 22, 2011. Accepted in final form August 31, 2011.

\section{REFERENCES}

1. O’Brien JT, Erkinjuntti T, Reisberg B, et al. Vascular cognitive impairment. Lancet Neurol 2003;2:89-98.

2. Neuropathology Group of the Medical Research Council Cognitive Function and Aging Study (MRC CFAS). Pathological correlates of late-onset dementia in a multicentre, community-based population in England and Wales. Lancet 2001;357:169-175.

3. Vermeer SE, Prins LD, den Heijer T, Hofman A, Koudstaal PJ, Breteler MM. Silent brains infarcts and the risk of dementia and cognitive decline. N Engl J Med 2003;348: 1215-1222.

4. van der Flier WM, van Straaten EC, Barkhof F, et al. Small vessel disease and general cognitive function in nondisabled elderly: the LADIS study. Stroke 2005;36:21162120 .

5. Jellinger KA, Attems J. Prevalence and pathogenic role of cerebrovascular lesions in Alzheimer disease. J Neurol Sci 2005;229-230:37-41.

6. Hachinski V, Iadecola C, Petersen RC, et al. National Institute of Neurological Disorders and Stroke-Canadian Stroke Network vascular cognitive impairment harmonization standards. Stroke 2006;37:2220-2241.

7. Kalaria RN, Kenny RA, Ballard CG, Perry R, Ince P, Polvikoski $\mathrm{T}$. Towards defining the neuropathological substrates of vascular dementia. J Neurol Sci 2004;226:75-80.

8. Gold G, Giannakopoulos P, Herrmann FR, Bouras C, Kovari E. Identification of Alzheimer and vascular lesion thresholds for mixed dementia. Brain 2007;130:2830-2836.

9. Perry R, Oakley A. Coronal map of Brodmann areas in the human brain. In: Roberts GLP, ed. Neuropsychiatric Disorders. London: Wolfe; 1993:1-10.

10. Ball MJ, Braak H, Coleman P, et al. Consensus recommendations for the postmortem diagnosis of Alzheimer's disease: The National Institute on Aging, and Reagan Institute Working Group on Diagnostic Criteria for the Neuropathological Assessment of Alzheimer's Disease. Neurobiol Aging 1997;18:S1-S2.

11. McKeith IG, Dickson DW, Lowe J, et al. Diagnosis and management of dementia with Lewy bodies: third report of the DLB Consortium. Neurology 2005;65:1863-1872.

12. Pantoni L, Sarti C, Alafuzoff I, et al. Postmortem examination of vascular lesions in cognitive impairment: a survey 
among neuropathological services. Stroke 2006;37:10051009.

13. Del Ser T, Bermejo F, Portera A, Arredondo JM, Bouras C, Constantinidis J. Vascular dementia: a clinicopathological study. J Neurol Sci 1990;96:1-17.

14. Zhu YC, Tzourio C, Soumare A, Mazoyer B, Dufouil C, Chabriat H. Severity of dilated Virchow-Robin spaces is associated with age, blood pressure, and MRI markers of small vessel disease: a population-based study. Stroke 2010;41:2483-2490.

15. De Reuck J, Deramecourt V, Cordonnier C, Leys D, Pasquier F, Maurage CA. Prevalence of small cerebral bleeds in patients with a neurodegenerative dementia: a neuropathological study. J Neurol Sci 2010;300:63-66.

16. Jellinger KA. The enigma of vascular cognitive disorder and vascular dementia. Acta Neuropathol 2007;113:349388.

17. Chui HC, Zarow C, Mack WJ, et al. Cognitive impact of subcortical vascular and Alzheimer's disease pathology. Ann Neurol 2006;60:677-687.

18. Ihara M, Polvikoski TM, Hall R, et al. Quantification of myelin loss in frontal lobe white matter in vascular dementia, Alzheimer's disease, and dementia with Lewy bodies. Acta Neuropathol 2010;119:579-589.

19. Rauramaa T, Pikkarainen M, Englund E, et al. Cardiovascular diseases and hippocampal infarcts. Hippocampus Epub 2010 Jan 6.

20. Vinters HV, Ellis WG, Zarow C, et al. Neuropathologic substrates of ischemic vascular dementia. J Neuropathol Exp Neurol 2000;59:931-945.

21. Gray F, Dubas F, Roullett E, Escourolle R. Leukoencephalopathy in diffuse hemorrhagic cerebral amyloid angiopathy. Ann Neurol 1985;18:54-59.
22. Kimberly WT, Gilson A, Rost NS, et al. Silent ischemic infarcts are associated with hemorrhage burden in cerebral amyloid angiopathy. Neurology 2009;72:1230-1235.

23. Pantoni L. Cerebral small vessel disease: from pathogenesis and characteristics to therapeutic challenges. Lancet Neurol 2010;9:689-701.

24. Pantoni L. Pathophysiology of age-related cerebral white matter changes. Cerebrovasc Dis 2002;13(suppl 2):7-10.

25. Esiri MM, Wilcock GK, Morris JH. Neuropathological assessment of the lesions of significance in vascular dementia. J Neurol Neurosurg Psychiatry 1997;63:749-753.

26. Polvikoski T, Van Straaten E, Barkhof F, et al. Frontal lobe white matter hyperintensities and neurofibrillary pathology in the oldest old. Neurology 2010;75:20712078.

27. Brun A. Pathology and pathophysiology of cerebrovascular dementia: pure subgroups of obstructive and hypoperfusive etiology. Dementia 1994;5:145-147.

28. Firbank MJ, He J, Blamire AM, et al. Cerebral blood flow by arterial spin labelling in post stroke dementia. Neurology 2011;76:1478-1484.

29. Savva GM, Wharton SB, Ince PG, Forster G, Matthews FE, Brayne C. Age, neuropathology, and dementia. N Engl J Med 2009;360:2302-2309.

30. White L. Brain lesions at autopsy in older JapaneseAmerican men as related to cognitive impairment and dementia in the final years of life: a summary report form the Honolulu-Asia aging study. J Alzheimers Dis 2009;18: 713-725.

31. Grinberg L, Heinsen $\mathrm{H}$. Toward a pathological definition of vascular dementia. J Neurol Sci 2010;299:136-138.

\section{Neurology ${ }^{\circledR}$ Launches Subspecialty Alerts by E-mail!}

Customize your online journal experience by signing up for e-mail alerts related to your subspecialty or area of interest. Access this free service by visiting http://www.neurology.org/site/subscriptions/ etoc.xhtml or click on the "E-mail Alerts" link on the home page. An extensive list of subspecialties, methods, and study design choices will be available for you to choose from-allowing you priority alerts to cutting-edge research in your field! 